\title{
Impact of cyclooxygenase- 2 over-expression on the prognosis of colorectal cancer patients
}

\author{
An experience from Western Saudi Arabia
}

Abdulkader M. Albasri, MBBCH, PhD, Mohammed A. Elkablawy, MSc, PhD, Akbar S. Hussainy, FCPS, PDFPath [Canada], Hala M. Yousif, MSc, MD, Ahmed S. Alhujaily, MBBCH, KSUFP.

\begin{abstract}
الأهداف : دراسة أثر زيادة مستوى بروتين (COX-2) على تطور

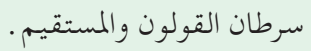

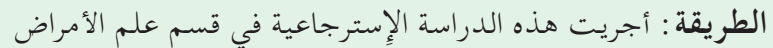

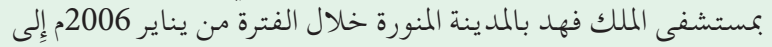

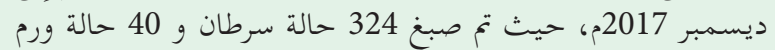
حميد و 20 حالة من نسيسج أمعاء طبيعي حين

النتائج : وقد اتضح زيادة مستوى البروتين في 40\% من الأنسجة

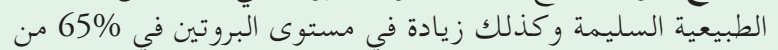

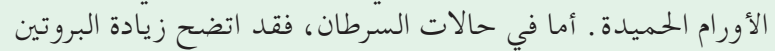

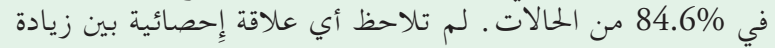

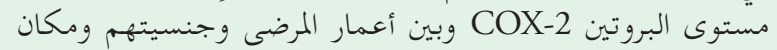

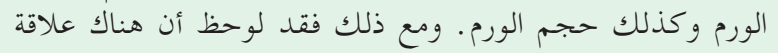

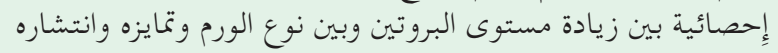

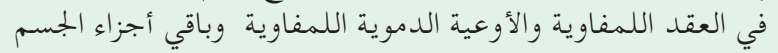

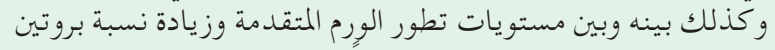

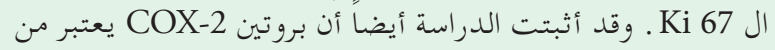

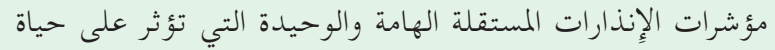

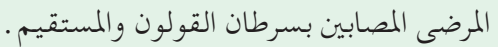

الحاتمة : وقد انتهت الدراسة إلى أن زيادة مستوى بروتين COX-2

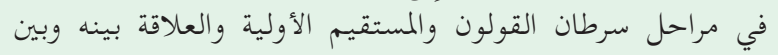

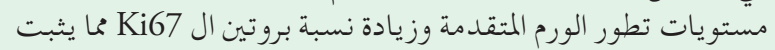

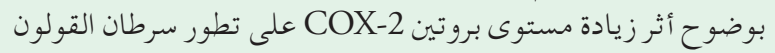
والمستقيم . بوضوح آنر

Objectives: To evaluate cyclooxygenase-2 (COX-2) overexpression in colorectal cancer (CRC) and its role in carcinogenesis and prognosis.

Methods: It was a retrospective study. Archival samples were obtained from Pathology Department at King Fahad Hospital, Madinah, Saudi Arabia, over 11 years' period (January 2006 to December 2017). Samples were analyzed using immunohistochemistry for COX-2 and Ki67 over-expression in 324 CRC patients, 40 cases of colorectal adenomas and 20 cases of normal colonic mucosa.

Results: Cyclooxygenase-2 over-expression was observed in $40 \%$ of normal colonic mucosa, $65 \%$ of colorectal adenoma and $84.6 \%$ of CRC cases. There were no significant correlations between COX-2 over-expression and age, gender, tumor site, or tumor size. However, COX-2 over-expression revealed highly significant correlations with tumor differentiation, lymph node metastasis, lympho-vascular invasion, distant metastasis, advanced stages, and high Ki67 expression. Univariate Kaplan-Meir survival analysis showed that patients with high COX-2 expression had significantly shorter periods of survival. Multivariate analysis by means of the COX2 regression model revealed that high COX-2 overexpression, AJCC, and Ki67 expression were the only significant independent prognostic indicators.

Conclusion: Cyclooxygenase-2 over-expression increases during normal-adenoma-carcinoma sequence, moreover COX-2 over-expression is associated with advanced tumor stage and Ki67 over-expression. These findings suggest a significant role of COX-2 in the carcinogenesis and prognosis of CRC in our study population.

Saudi Med J 2018; Vol. 39 (8): 773-780 doi:10.15537/smj.2018.8.22837

From the Department of Pathology, Taibah University, Madinah, Kingdom of Saudi Arabia.

Received 29th April 2018. Accepted 13th July 2018.

Address correspondence and reprint request to: Dr. Abdulkader M. Albasri, Head of Pathology Department, c: abdbasri@hotmail.com 
$\mathrm{C}$ olorectal cancer (CRC) is one of the most common cancers in the world today, being second most common in the female population and third in the males. More than half [55\%] cases of CRC occur in the developed countries. The incidence of CRC is very low in Asian and African countries. Although there is a great geographical variation in the incidence across the globe; the geographical pattern is similar within the 2 genders. ${ }^{1}$ The Kingdom of Saudi Arabia (KSA) belongs to low CRC incidence region; CRC is still the second common, after, according to the latest Saudi cancer registry. ${ }^{2}$ Analysis of KSA CRC suggests a potentially disturbing rise in CRC in near future, but no formal surveillance programs have yet been established in the Kingdom. ${ }^{3}$ In the latest hospital and pathology-based study from the region of Al-Madinah, most of the CRC patients were diagnosed in an advanced stage. ${ }^{4}$ There is a well-established link between chronic inflammation and carcinogenesis. Several inflammatory mediators and cytokines appear to be involved in this pathogenesis, one of them being the family of cyclooxygenases. These are the enzymes involved in prostaglandin biosynthesis; the isoform cyclooxygenase-2 (COX-2) is found to be up regulated in inflammations and cancers. ${ }^{5}$ The overexpression of COX-2 is associated with poor prognosis in CRC patients. The growth and progress of CRC has been reported to accompany COX-2 gene overexpression. High levels of COX-2 over-expression were more frequent in early colorectal lesions. Furthermore, COX-2 over-expression in the normal tissue adjacent to the tumor may also be involved in the tumorigenesis of CRC. Thus, these interpretations seem to be useful in defining the importance of COX-2 over-expression in the tumorigenesis of CRC. ${ }^{6}$ Cyclooxygenase- 2 over-expression appears to provide useful prognostic information for CRC, while predicting the patients who are at a higher risk for recurrence. ${ }^{7}$ However, the prognostic value of COX-2 over-expression on CRC patient survival has yet to be established using large-scale prospective trials. ${ }^{8,9}$

The aim of this study is to immunohistochemically examine the COX-2 biomarker in CRC patients and to correlate the COX-2 findings with clinicopathological prognostic parameters and the proliferative marker Ki67. This study is significant because no similar study has been performed in our region in KSA, and such a

Disclosure. Authors have no conflict of interests, and the work was not supported or funded by any drug company. study would be helpful in establishing any predictive or prognostic role of COX-2 in managing CRC patients within the KSA population.

Methods. Generic search engines namely Google and MSN Bing search engines were utilized; as well as specific medical search engines Pubmed/Medline, Science Direct, Scopus and Google scholar were used for search of preceding scientific literature research. The present study was a retrospective study involving the archival histopathology material and record; and did not involve patients' personal information or any implication upon the management; thus, was according to the principles of Helsinki Declaration. Hence no ethical approval was mandatory in the present study.

This retrospective study included 324 consecutive cases of CRC diagnosed at the Pathology Department, King Fahad Hospital, Madinah, Saudi Arabia, over a period of 11 years (January 2006 to December 2017). Clinicopathological data were obtained from patients' medical records and included gender, age, tumor type, size, site, grade, lymphovascular invasion, lymph nodes status, American Joint Committee on Cancer (AJCC) stages of CRC, and Ki67 immunostaining results.

In the present study, 40 colorectal adenoma cases and 20 normal colonic mucosa cases were used as controls. The inclusion criterion was histologically confirmed invasive CRC cases. Whereas the exclusion criteria were in-situ lesions, recurrences, biopsies, benign lesions, and metastatic lesions. All total colectomies, hemicolectomies, and excision specimens were included in the analysis.

Immunohistochemical procedures. Cores of sample tissues were extracted from archival paraffin blocks of CRC and utilized in the construction of tissue miniarray (TmA), as previously described. ${ }^{10}$ Fourmicrometer-thick tissue sections were cut from the TmA blocks, mounted on charged poly-l-lysine-coated slides, and followed by immunohistochemistry (IHC) using the Avidin Biotin detection system according to the manufacturer's instructions. The antibody used was a human anti-COX-2 monoclonal antibody (diluted in 1:50 blocking solution; Clone 4A4, Ventana Inc., Tucson, AZ, United States). Sections were doublestained with another antibody (anti-Ki67; diluted 1:100, in blocking solution; Ventana Inc., Tucson, AZ, United States). Immunohistochemistry was carried out by an automatic immune-stainer (Ventana Bench Mark XT; Ventana Inc., Tucson, AZ, United States). Sample of normal prostatic tissue was used as a positive control for COX-2. Negative controls were accomplished by replacing the primary antibody with serum. 
Interpretation of immunohistochemical staining. Distinct yellow to brown staining of cellular cytoplasm was considered positive for COX-2. The authors (AMA and MAE) reviewed the immunostaining independently and the average score was taken. The level of COX-2 over-expression was calculated by combining an estimate of the percentage of immuno-reactive cells (quantity score) with an estimate of the staining intensity (staining intensity score). No staining was scored as $0,1-10 \%$ of cells with positive staining were scored as $1,10-50 \%$ as $2,50-70 \%$ as 3 , and $70-100 \%$ as 4 . Staining intensity was rated on a scale of 0 to 3 as follows: $0=$ negative (no staining); 1 = weak, 2 = moderate, and $3=$ strong. The final expression score was expressed as follows: '-' for scores 0, ' + ' for scores 1-3, '++' for scores 4-6 and ' +++ ' for scores $>6$. For statistical analysis, we combined the cases that scored as '-' and ' + ' (low score) and compared them to the cases that scored as ' ++ ' and ' +++ ' (high score).

Statistical analysis. Data analysis was performed by utilizing the Statistical Package for Social Sciences (SPSS) Version 22.0 for Windows (IBM Corp, Armonk, NY, USA). Correlation analysis between the 2 data sets was used to assess the inter observer reproducibility. Chi-squared and Fisher's exact tests were performed to determine the association between the COX-2 expression and patient clinicopathological parameters. The cumulative patient survival was assessed by the Kaplan-Meier method. Finally, the long-rank test was used for comparison of the survival curves. The COX-2 proportional hazard linear regression model was performed by forward stepwise procedure, to look for the independent factors independently associated with survival. A $p$ value of $\leq 0.05$ was considered significant for all statistical analyses.

Results. Clinicopathological characteristics of the cases. A total of 324 patients were included in the study. There were 196 cases (60.5\%) males and 128 cases $(39.5 \%)$ females giving a male to female ratio of 1.5:1. The age of the studied cases ranged from 22 to 96 years with a mean age 56.9 years. Left-sided tumors were more common than the right-sided and seen in $63.5 \%$ of cases. Tumor size was $\geq 4 \mathrm{~cm}$ in $84 \%$ of the studied cases. The most common histologic type was adenocarcinoma, seen in 279 cases (86.1\%). Most of tumors were moderately differentiated (80.2\%). The majority of patients were diagnosed in stage II $(44.7 \%)$ and stage III (31.5\%) according to the AJCC. Approximately $44.8 \%$ of cases had positive lymph node metastasis and lymphovascular invasion, where distant metastasis were seen in $30.3 \%$ of cases.
Table 1 - The clinicopathological features of 324 colorectal cancer (CRC) cases.

\begin{tabular}{|c|c|c|}
\hline Variables & $\mathrm{n}$ & $(\%)$ \\
\hline \multicolumn{3}{|l|}{ Age (years) } \\
\hline$<40$ & 177 & $(54.6)$ \\
\hline$\geq 40$ & 147 & $(45.4)$ \\
\hline \multicolumn{3}{|l|}{ Gender } \\
\hline Female & 128 & $(39.5)$ \\
\hline Male & 196 & $(60.5)$ \\
\hline \multicolumn{3}{|l|}{ Tumor site } \\
\hline Colon & 168 & $(51.9)$ \\
\hline Rectum & 156 & $(48.1)$ \\
\hline \multicolumn{3}{|l|}{ Tumor size } \\
\hline$<4 \mathrm{~cm}$ & 52 & $(16.0)$ \\
\hline$\geq 4 \mathrm{~cm}$ & 272 & $(84.0)$ \\
\hline \multicolumn{3}{|l|}{ Tumor type } \\
\hline Adenocarcinoma & 279 & $(86.1)$ \\
\hline Mucinous adenocarcinoma & 45 & $(13.9)$ \\
\hline \multicolumn{3}{|l|}{ Tumor differentiation } \\
\hline Well & 49 & $(15.1)$ \\
\hline Moderate & 260 & $(80.2)$ \\
\hline Poor & 15 & $(4.7)$ \\
\hline \multicolumn{3}{|l|}{ Lymph node } \\
\hline Negative & 179 & $(55.2)$ \\
\hline Positive & 145 & $(44.8)$ \\
\hline \multicolumn{3}{|l|}{ Lymphovascular invasion } \\
\hline Negative & 179 & $(55.2)$ \\
\hline Positive & 145 & $(44.8)$ \\
\hline \multicolumn{3}{|l|}{ Distant metastasis } \\
\hline Negative & 281 & $(86.7)$ \\
\hline Positive & 43 & $(13.3)$ \\
\hline \multicolumn{3}{|l|}{ AJCC } \\
\hline I & 34 & $(10.5)$ \\
\hline II & 145 & $(44.7)$ \\
\hline III & 102 & $(31.5)$ \\
\hline IV & 43 & (13.3) \\
\hline
\end{tabular}

Table 1 summarizes the clinicopathological characteristics of 324 CRC cases.

Cyclooxygenase-2 over expression profiles. In the normal colonic mucosa, COX-2 over-expression was observed in $40 \%$ cases ( 8 of 20 cases); whereas in colorectal adenoma samples, COX-2 was over-expressed in $65 \%$ cases (26 of 40 cases). In CRC, COX-2 expression was high in most cases namely, 274 cases (84.6\%), and low in only 50 cases (15.4\%) (Figure 1). Similarly, a higher COX-2 over expression was observed in high-grade villous adenomas as compared to low-grade tubular adenomas $(p=0.081)$.

Correlation of COX-2 expression with the clinicopathological features. Cyclooxygenase-2 overexpression did not show any significant correlation when compared with age, gender, tumor site and tumor 
Table 2 - Correlation of COX-2expression with the clinicopathological variables and Ki67 immunophenotyping of colorectal cancer (CRC) cases.

\begin{tabular}{|c|c|c|c|}
\hline Variables & $\begin{array}{c}\text { Low } \\
\text { COX-2 }\end{array}$ & $\begin{array}{c}\text { High } \\
\text { COX-2 }\end{array}$ & $P$-value \\
\hline Age (years) & & & 0.287 \\
\hline$<40$ & 25 & 152 & \\
\hline$\geq 40$ & 25 & 122 & \\
\hline Gender & & & 0.11 \\
\hline Female & 12 & 116 & \\
\hline Male & 38 & 158 & \\
\hline Tumor site & & & 0.183 \\
\hline Colon & 26 & 142 & \\
\hline Rectum & 24 & 132 & \\
\hline Tumor size & & & 0.217 \\
\hline$<4 \mathrm{~cm}$ & 28 & 145 & \\
\hline$\geq 4 \mathrm{~cm}$ & 22 & 129 & \\
\hline Tumor type & & & 0.026 \\
\hline Adenocarcinoma & 38 & 241 & \\
\hline Mucinous adenocarcinoma & 12 & 33 & \\
\hline Tumor differentiation & & & $<0.001$ \\
\hline Well & 19 & 30 & \\
\hline Moderate & 31 & 229 & \\
\hline Poor & 0 & 15 & \\
\hline Lymph node & & & 0.001 \\
\hline Negative & 38 & 141 & \\
\hline Positive & 12 & 33 & \\
\hline Lymphovascular invasion & & & 0.001 \\
\hline Negative & 38 & 141 & \\
\hline Positive & 12 & 33 & \\
\hline Distant metastasis & & & $<0.001$ \\
\hline Negative & 50 & 231 & \\
\hline Positive & 0 & 43 & \\
\hline AJCC stage & & & $<0.001$ \\
\hline I & 19 & 15 & \\
\hline II & 19 & 126 & \\
\hline III & 12 & 90 & \\
\hline IV & 0 & 43 & \\
\hline Ki67 & & & $<0.001$ \\
\hline Low & 23 & 39 & \\
\hline High & 27 & 235 & \\
\hline COX-2 - cyclooxygenase- 2 & $\begin{array}{l}\text { CC - Ame } \\
\text { ancer }\end{array}$ & int $\mathrm{Cor}$ & tee on \\
\hline
\end{tabular}

size. On the contrary, COX-2 over-expression exhibited a significant correlation with tumor type $(p=0.026)$; whereas highly significant correlations were seen with tumor differentiation $(p<0.001)$, positive lymph node metastasis $(p<0.001)$, lympho-vascular invasion $(p<0.001)$, distant metastasis $(p<0.001)$, advanced AJCC stages $(\mathrm{p}<0.001)$, and high Ki67 expression $(p<0.001)$. Figure 2 depicts the association between increased COX-2 immunoexpression and increased Ki67 immunoexpression. The summary of correlation of COX-2 over-expression with clinicopathological variables depicted in Table 2.
Table 3 - Test statistics for equality of survival distribution for prognostic factors examined in 324 colorectal carcinomas. A univariate approach to cancer-specific mortality.

\begin{tabular}{lccc}
\hline Factor & $\begin{array}{c}\text { Univariate } x^{2} \\
\text { for the } \\
\text { log-rank }\end{array}$ & $\begin{array}{c}\text { Degree of } \\
\text { freedom }\end{array}$ & P-value \\
\hline Clinicopathological factors & 772.81 & 3 & $<0.001$ \\
AJCC stage & 400.28 & 1 & $<0.001$ \\
Lymph node stage & 507.58 & 1 & $<0.001$ \\
Metastasis stage & 400.28 & 1 & $<0.001$ \\
Lymphovascular invasion & 170.27 & 2 & $<0.001$ \\
Histological tumor grade & 2.141 & 1 & 0.133 \\
Tumor size & 2.360 & 1 & 0.212 \\
Tumor site & 1.153 & 1 & 0.283 \\
Tumor type & 0.308 & 1 & 0.579 \\
Age & 0.064 & 1 & 0.800 \\
Gender & & & $<0.001$ \\
Immunostaining & 71.67 & 1 & $<0.001$ \\
Cox2 immunostaining & 23.91 & 1 & \\
Ki67 immunostaining & Cancer & & \\
\hline COX-2 - cyclooxygenase-2, AJCC - American Joint Committee on &
\end{tabular}

Univariate and multivariate long-term survival analysis. All the significant features associated with survival $(p<0.05)$ for the 324 CRC patients in the study are depicted in Table 3 . Figure 3 exhibit significant survival curves for COX-2 immunoprofile. Significantly short survival was observed in patients with high COX-2 over-expression than the patients with low COX-2 over-expression $(p<0.001)$. High COX-2 expression $(p<0.001)$, AJCC $(p<0.001)$ and Ki67 expression $(p<0.014)$ were the only significant independent prognostic indicators, as calculated by multivariate analysis of COX regression model.

Discussion. Cyclooxygenase is a rate-limiting enzyme in the prostaglandin metabolism and has 2 isoforms COX-1 and COX-2. The extensively studied isoform COX-2 is the enzyme, which is up regulated in response to cytokines, growth factors and several tumorpromoting factors. Its pathophysiological role has been well recognized in inflammations, healing and repair as well as in carcinogenesis. The carcinogenetic effects of COX-2 have been studied in cancers of various organs such as breast, ${ }^{11}$ lung, ${ }^{12}$ esophagus, ${ }^{13}$ head and neck,,${ }^{14}$ bladder, ${ }^{15}$ and liver. ${ }^{16}$ Cyclooxygenase- 2 has also been found to be over-expressed in CRC., ${ }^{7,9,17}$ This role of COX-2 in the human malignancies, including CRC was discovered by recent epidemiological studies, which reported an approximately $50 \%$ decrease in the relative risk of CRC in individuals using non-steroidal antiinflammatory drugs; suggesting that these drugs might have protective and therapeutic effects. ${ }^{18}$ 


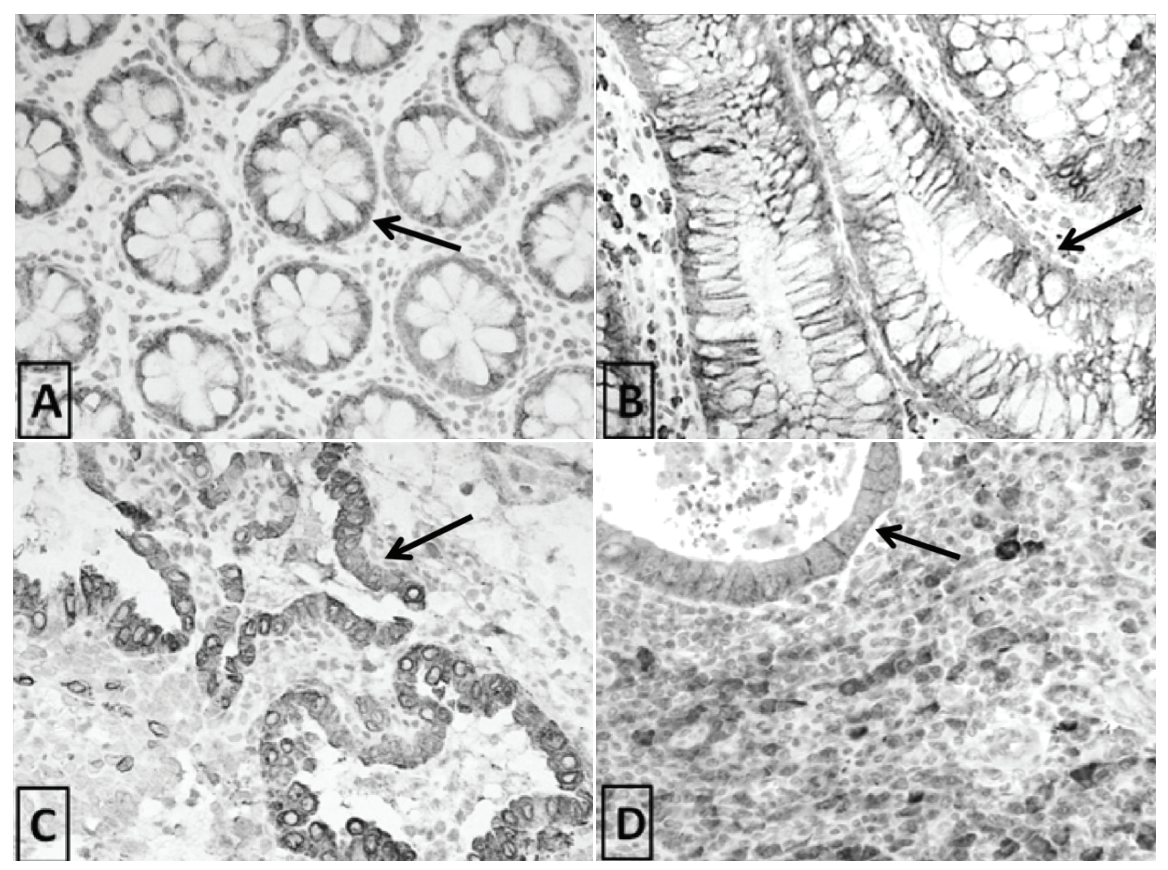

Figure 1 - Different patterns of immunohistochemical COX-2 expression in A) normal colon (weak cytoplasmic positivity), B)colonic adenoma (mild to moderate cytoplasmic positivity), C) colorectal carcinoma (marked granular cytoplasmic expression), and D) lymph node metastasis CRC (marked granular cytoplasmic expression). Arrows indicate COX-2 cytoplasmic expression.

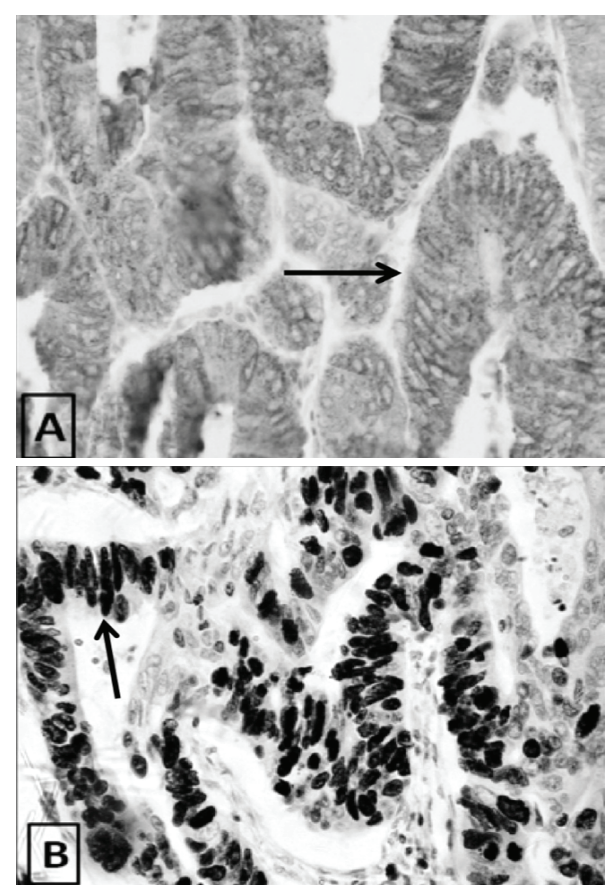

Figure 2 - Immunohistochemical characterization of colorectal cancer specimens showing high levels of Ki67 expression confirming the association between increased $(+++)$ COX-2 immunoexpression and increased $(+++)$ Ki67 immunoexpression. Arrows indicate COX-2 cytoplasmic expression and $\mathrm{Ki} 67$ nuclear expression.

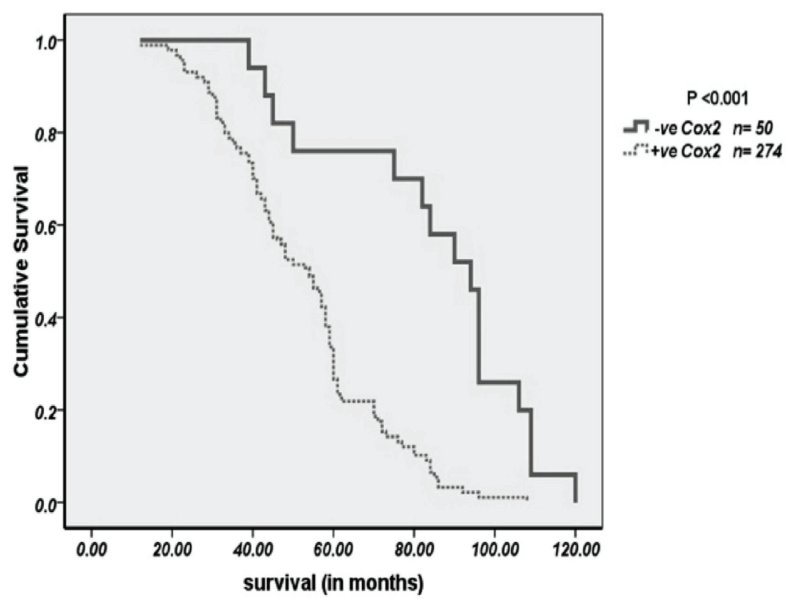

Figure 3 -Shows Kaplan-meier curves for significant overall survival functions for Cox2 expression in CRC.

In this study, we found that COX-2 expression was up regulated in the sequence from normal to adenoma to adenocarcinoma, in concordance with the findings of a number of previous studies suggesting the pathogenic role of COX-2 in CRC. ${ }^{19}$ However, the over expression of COX-2 in the adjacent normal mucosa had been an important point of discussion in the past. Some studies reported a higher expression of COX-2 in adjacent 
normal mucosa as compared to that of CRC mucosa; ${ }^{20}$ whereas in contrast, some studies have observed absence of COX-2 expression in the normal mucosa near the CRC. ${ }^{21}$ Recently Lin et $\mathrm{al}^{6}$ from Taiwan, have studied the COX-2 expression in the CRC and normal mucosa, through IHC and western blotting methodologies. They observed that most of their patients had low levels of COX-2 expression in CRC tissue and high levels of COX-2 expression in adjacent normal mucosa. These observations confirm our findings of COX-2 expression in normal mucosa; although we found higher tumor to normal mucosa expression ratio of COX-2. Lin et $\mathrm{al}^{6}$ observed a high ratio of tumor to normal tissue COX-2 expression only in correlation with high recurrence rates and poor prognosis; we found the similar correlation with higher tumor stage. Thus, we agree with their conclusion that COX-2 expression in normal tissue adjacent to tumors may be important for CRC carcinogenesis.

The role of COX-2 expression and its inhibitors have also been studied in depth for its association with the colorectal adenoma, in an attempt to prove its carcinogenetic importance. Recently, a review on COX-2 over-expression and chemoprevention of CRC, report four randomized studies, which have shown that daily aspirin use reduces the occurrence of colorectal adenomatous polyps among individuals with a history of prior colorectal adenomas or cancer. ${ }^{22}$ In the same year, Wasilewicz et $\mathrm{al}^{23}$ demonstrated that high COX-2 expression is associated with distinctive risk factors in the process of malignant transformation of colonic polyps. ${ }^{23}$ In contrast, a group of scientists from Brazil, in a study using TmA and IHC on positivity and intensity of COX-2 did not find any significant differences between the adenomas and the non-neoplastic colorectal mucosa. ${ }^{24}$ Our findings in the present study are however in concordance with most of the recent works regarding its over-expression and its significance as precancerous marker in the colorectal adenoma-carcinoma sequence. We observed a trend of COX-2 over-expression in high-grade villous adenomas as compared to low-grade tubular adenomas. Our findings are in concordance with a recent study reporting statistically significant differences in high COX-2 expressions in high-grade dysplastic adenomas versus adenomas without dysplasia. They also report similar significant differences between low-grade dysplastic adenomas versus adenomas without dysplasia. ${ }^{23}$ In 2010, a large study by Benamouzig et al, reported that over-expression of COX-2 was frequent and predominated in large and high-grade dysplastic adenomas. ${ }^{25}$ Yet another study on colorectal polypi observed that COX-2 over-expression is infrequent in hyperplastic polyp, sessile serrated polyp/adenomas (SSA) and mixed polypi, as compared to non-serrated and serrated adenoma. Cyclooxygenase-2 overexpression becomes more frequent as tumors progress to higher-grades. ${ }^{26}$

In contrast to normal mucosa and adenoma samples, we found very high COX-2 expression in CRC samples, which correlated with higher stage tumors in our patient cohort. Kazem et al, ${ }^{7}$ from Egypt have recently reported statistically significant relationships between pathological grade and COX-2 positivity scores. Dimberg et $\mathrm{al}^{27}$ from Sweden also report COX-2 over-expression in CRC, however they observed this over-expression to be significantly higher in rectal carcinomas as compared to other locations of colon, probably due to differences in gene regulatory factors affecting COX-2 expression. Our observations are also in concordance with that of similar study on the carcinogenetic role of COX-2.28 No correlation was observed between COX-2 over- expression versus gender, age, tumor size, tumor and tumor location; similarly, Chinese scientists in their COX-2 study in $\mathrm{CRC}$ also could not find any correlation between the over-expression of COX-2 with gender, age, or tumor location. ${ }^{29}$ Likewise, Egyptian group of scientists, found no significant correlation between COX-2 overexpression and clinicopathological parameters, such as age, gender, tumor location. ${ }^{7}$ From within the KSA, similar were the observations by a group of researchers namely, they did not find any significant correlations between COX-2 over-expression and gender, age, grade or tumor location. ${ }^{8}$ However, in our study, a significant correlation of COX-2 over-expression with lymph node metastasis, distant metastasis and advanced AJCC stage, as observed by the Saudi group of scientists. ${ }^{8}$ Moreover, a highly significant association was observed between over expression of COX-2 and high expression of the proliferative marker ki67, which is in concordance with a number of previous studies. ${ }^{30-32}$ Thus, our findings concerning the COX-2 over-expression and clinicopathological parameters are in keeping with that of similar studies from the world and nation.

Previous studies have reported a direct relationship between COX-2 over-expression and patient survival in CRC. Our results in univariate and multivariate survival analysis support the theory that COX-2 over-expression is associated with a worse patient survival as compared to COX-2 negativity. Similar results have been reported and supported the prognostic role of COX-2 in CRC patients. ${ }^{8,30}$ 
In conclusion, we studied COX-2 in our CRC patients through TmA and IHC techniques, and found increasing percentage of COX-2 over-expression from normal to adenoma to carcinoma sequence. Similarly, a trend of high COX-2 over-expression was observed in high-grade villous adenomas as compared to low-grade tubular adenomas. Moreover, we observed significant correlations for advanced AJCC stage, as well as for increased expression of Ki67. All these findings suggest a significant role of COX-2 expression in carcinogenesis and progression of CRC. Thus, we confirm that COX-2 and Ki67 over-expression can be used as a reliable indicator for prognosis of CRC patients.

Acknowledgment. The authors would like to thank the Deanship of Scientific Research, Taibah University, Almadinah Almonawwarah, Saudi Arabia, for their encouragement and support. The authors would also like to thank Dr. Alla Khadri (Pathology Department, Taibah University, Saudi Arabia) for revising the grammar and language. And also thank to Mr. Mohamed Abdelsammad, Mr. Rayid Alharbi, and Mrs. Faten Bokhary (laboratory technician, Taibah University, Saudi Arabia) for technical advice. Furthermore, we would like to thank Scribendi, the Editing and Proofreading Services for English Documents (https://www.scribendi.com/) for the English language editing of the manuscript.

\section{References}

1. Ferlay J, Soerjomataram I, Dikshit R, Eser S, Mathers C, Rebelo M, et al. Globocan 2012 v1.0, Cancer Incidence and Mortality Worldwide: IARC Cancer Base No. 11 [Internet]. [2015 November 2015]. Lyon (FR): International Agency for Research on Cancer; 2013.

2. International Association of Cancer Registries. Saudi Cancer Incidence Report 2014. Saudi Cancer Registry. Riyadh (KSA): King Faisal Specialist Hospital \& Research Center Council of Health Services; 2017.

3. Ibrahim EM, Zeeneldin AA, El-Khodary TR, Al-Gahmi AM, Bin Sadiq BM. Past, Present and Future of Colorectal Cancer in the Kingdom of Saudi Arabia. Saudi J Gastroenterol 2008; 14: 178-182.

4. Albasri A, Yosef H, Hussainy AS, Sultan SA, Alhujaily AS. Histopathological features of colorectal cancer in Al-Madinah region of Saudi Arabia: 8 years experience. Asian Pac J Cancer Prev 2014; 15: 3133-3137.

5. Sobolewski C, Cerella C, Dicato M, Ghibelli L, Diederich M. Role of Cyclooxygenase-2 in cell proliferation and cell death in human malignancies. Int J Cell Biol 2010; 2010: 215158.

6. Lin PC, Lin YJ, Lee CT, Liu HS, Lee JC. Cyclooxygenase-2 expression in the tumor environment is associated with poor prognosis in colorectal cancer patients. Oncol Lett 2013; 6: 733-739.

7. Kazem A, El Sayed K, El Kerm Y. Prognostic significance of COX-2 and $\beta$-catenin in colorectal carcinoma. Alexandria Journal of Medicine 2014; 50: 211-220.

8. Al-Maghrabi J, Buhmeida A, Emam E, Syrjänen K, Sibiany A, Al-Qahtani M, et al. Cyclooxygenase-2 expression as a predictor of outcome in colorectal carcinoma. World J Gastroenterol 2012; 18: 1793-9.
9. Peng L, Zhou Y, Wang Y, Mou H, Zhao Q. Prognostic significance of COX-2 immunohistochemical expression in colorectal cancer: a meta-analysis of the literature. PLoS One 2013; 8: e58891.

10. Elkablawy MA, Albasri AM. High quality tissue miniarray technique using a conventional TV/radio telescopic antenna. Asian Pac J Cancer Prev 2015; 16: 1129-1133.

11. Denkert C, Winzer KJ, Hauptmann S. Prognostic impact of cyclooxygenase-2 in breast cancer. Clin Breast Cancer 2004; 4: 428-433.

12. Mascaux C, Martin B, Paesmans M, Berghmans T, Dusart M, Haller A, et al. Has COX-2 a prognostic role in non-small-cell lung cancer? A systematic review of the literature with metaanalysis of the survival results. Br J Cancer 2006; 95: 139-145.

13. Li L, Zhao J, Wu Z, Wang G, Chen G. Meta-analysis: clinicopathological and prognostic significance of cyclooxygenase-2 expression on oesophageal squamous cell carcinoma. Aliment Pharmacol Ther 2009; 30: 589-596.

14. Lin DT, Subbaramaiah K, Shah JP, Dannenberg AJ, Boyle JO. Cyclooxygenase-2: a novel molecular target for the prevention and treatment of head and neck cancer. Head Neck 2002; 24 : 792-799.

15. Hammam OA, Aziz AA, Roshdy MS, Abdil Hadi A. Possible role of cyclooxygenase- 2 in schistosomal and non-schistosomal associated bladder cancer. Medscape J Med 2008; 10: 60.

16. Bayomi EA, Barakat AB, El-Bassuoni MA, Talaat RM, El-Deftar MM, Abdel Wahab SA, etal. Cyclooxygenase-2 expression is associated with elevated aspartate aminotransferase level in hepatocellular carcinoma. J Cancer Res Ther 2015; 11 : 786-792.

17. Brown JR, DuBois RN. COX-2: a molecular target for colorectal cancer prevention. J Clin Oncol 2005; 23: 2840-2855.

18. Cooper K, Squires H, Carroll C, Papaioannou D, Booth A, Logan RF, et al. Chemo prevention of colorectal cancer: systematic review and economic evaluation. Health Technol Assess 2010; 14: 1-206.

19. Arber N, Eagle CJ, Spicak J, Rácz I, Dite P, Hajer J, et al. Celecoxib for the prevention of colorectal adenomatous polyps. N Engl J Med 2006; 355: 885-895.

20. Charalambous MP, Lightfoot T, Speirs V, Horgan K, Gooderham NJ. Expression of COX-2, NF-kappaB-p65, NF-kappaB-p50 and IKKalpha in malignant and adjacent normal human colorectal tissue. Br J Cancer 2009; 101: 106-115.

21. Sano H, Kawahito Y, Wilder RL,Hashiramoto A, Mukai S, Asai K, et al. Expression of cyclooxygenase-1 and -2 in human colorectal cancer. Cancer Res 1995; 55: 3785-3789.

22. Chan AT. COX-2 expression in adenoma: an imperfect marker for chemoprevention. Gut 2010; 59: 568-569.

23. Wasilewicz MP, Kołodziej B, Bojułko T Kaczmarczyk M, Sulzyc-Bielicka V, Bielicki D. Expression of cyclooxygenase-2 in colonic polyps. Pol Arch Med Wewn 2010; 120: 313-320.

24. Nogueira RB, Pires AR, Soares TM, Rodrigues SR, Campos MA, Toloi GCet al. Immunoexpression of the COX-2, p53, and caspase-3 proteins in colorectal adenoma and non-neoplastic mucosa. Einstein 2013; 11: 456-461.

25. Benamouzig R, Uzzan B, Martin A. Cyclooxygenase-2 expression and recurrence of colorectal adenomas: effect of aspirin chemo prevention. Gut 2010; 59: 622-629. 
26. Kawasaki T, Nosho K, Ohnishi M. Cyclooxygenase-2 overexpression is common in serrated and non-serrated colorectal adenoma, but uncommon in hyperplastic polyp and sessile serrated polyp/adenoma. BMC Cancer 2008; 8: 33.

27. Dimberg J, Samuelsson A, Hugander A. Differential expression of cyclooxygenase 2 in human colorectal cancer. Gut 1999; 45: 730-732.

28. Dixon DA, Blanco FF, Bruno A. Mechanistic Aspects of COX-2 Expression in Colorectal Neoplasia. Recent Results In Cancer Research 2013; 191: 7-37.

29. Wu QB, Sun GP. Expression of COX-2 and HER-2 in colorectal cancer and their correlation. World J Gastroenterol 2015; 21 : 6206-6214.
30. Jafarian AH, Kermani AT, Esmaeili J.The role of COX-2 and $\mathrm{Ki}-67$ over-expression in the prediction of pathologic response of rectal cancer to neoadjuvant chemoradiation therapy. Indian J Cancer 2016; 53: 548-551.

31. Güler SA, Uğurlu MU, Kaya H, Şen S, Nazlı Y, M, Güllüoğlu BM. Impact of cyclooxygenase-2 over-expression on the prognosis of breast cancer patients. Güllïoğlu Ulus Cerrahi Derg 2016; 32: 81-88.

32. Joo Y, Chung I, Park Y,Koh YS, Lee JH, Park CH, et al.Expression of Cyclooxygenase-2, p53 and Ki-67 in Gastric Cancer. J Korean Med Sci 2006; 21: 871-876. 